\title{
WAHRHEIT ALS FREIHEIT JAMES MENSCH ÜBER DAS VERHÄLTNIS VON BEWEGUNG UND RECHT BEI PATOČKA ${ }^{1}$
}

\author{
HANS RAINER SEPP
}

\begin{abstract}
In his book Patočka's Asubjective Phenomenology: Toward a New Concept of Human Rights, published in 2016, James Mensch analyses Jan Patočka's phenomenology of the movement of human existence, showing the extent to which Patočka follows European philosophical traditions, above all Aristotle, and interprets central concepts like movement, existence, essence, body and soul in a new way. Mensch explains that for Patočka, the care for the manifestations of existence in the context of its movements as the ontological framework for human rights will only be fulfilled under the condition that in the public space of mutual understanding, the rights of free speech and free exchange are guaranteed and the single existence can realise his or her explicit freedom that is at the same time always related to and dependent on the Other. This result lays the ground for further questions around the relationship of care and right within an intercultural context, in particular in regard to basic characteristics of law practices in the life-world of Australian Aborigines.
\end{abstract}

\section{James Mensch über Patočka}

In seiner Auslegung von Patočkas Phänomenologie der Bewegung der menschlichen Existenz zeigt James Mensch, ${ }^{2}$ wie sich Patočka an die europäische

1 Die vorliegende Publikation ist an der Humanwissenschaftlichen Fakultät der Karls-Universität Prag im Rahmen des Forschungsvorhabens Life and Environment: Phenomenological Relations between Subjectivity and Natural World (Czech Science Foundation, GA ČR, n. 15-10832S) entstanden.

2 Mensch James, Patočka's Asubjective Phenomenology: Toward a New Concept of Human Rights, Würzburg, Königshausen \& Neumann, Orbis Phaenomenologicus: Studien 38, 2016.

https://doi.org/10.14712/24646504.2018.9

(C) 2017 The Author. This is an open-access article distributed under the terms of the

Creative Commons Attribution License (http://creativecommons.org/licenses/by/4.0). 
Denktradition, insbesondere an Aristoteles, anlehnt und wie dabei zentrale Begriffe und ihr gedanklicher Zusammenhang eine Neuorientierung erfahren. Die Begriffe, um die es dabei vor allem geht, sind Bewegung, Existenz und Essenz, Seele und Leib sowie Freiheit. ${ }^{3}$

Den grundlegenden Begriff der Bewegung versteht Patočka, so Mensch, im Anschluss an Aristoteles als Aktualisierung ( $\dot{v} v \tau \lambda \lambda \dot{\varepsilon} \chi \varepsilon ı \alpha)$ im Sinne eines Prozesses des Heraus- und Hervortretens. Die Auffassung einer engen Zusammengehörigkeit von Seele und Leib orientiere sich ebenfalls an Aristoteles: ,Seele' als verleiblichtes, sensuelles Organ bezeichne für Patočka die Bewegung menschlicher Existenz, nämlich die Aktualisierung des lebendigen Körpers im Prozess seines Sich-Vollziehens

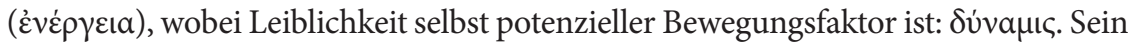
,Ich kann' besagt nicht die Absicht eines Subjekts, sondern Existenz findet sich mit dem ,Ich kann' ihrer Leiblichkeit in die Mitte von Welt versetzt: als das erfahrende Zentrum einer horizonthaften Auffaltung von Welt.

Was die Freiheit betrifft, so bestimmt Mensch sie mit Blick auf Patočka als grundlegendes Merkmal der Bewegung menschlicher Existenz: Als solches sei sie diejenige Bewegung, welche die verleiblichte Seele mit ihrem Hervortreten vollzieht. Ist das hinausstehende Hervortreten Raum einnehmend und Zeit stiftend, so überschreitet es damit das unmittelbar Gegebene eines räumlichen Hier und zeitlichen Jetzt; es verfährt in diesem Sinn distanzierend, Distanz schaffend, und dieses Distanzieren mittels raum-zeitlicher Ekstasen ist die Basis von Freiheit. Mit der Freiheit seiner Distanznahme verhält sich menschliche Existenz zum Abwesenden, zu dem, was nicht (hier und jetzt) ist, so dass ,Freiheit' als ein je bestimmter Stil der Bewegung menschlicher Existenz im Verhältnis zu dem, was nicht anwesend ist, gefasst werden kann. Dies ist zugleich Ausdruck menschlicher Endlichkeit, da, wie Mensch betont, Freiheit in diesem ursprünglichen Sinn die Befähigung besagt, eine Bandbreite möglicher Wirklichkeiten zu imaginieren, von der ein Leben nur einen Teil tatsächlich verwirklichen kann. Daraus folge: Um diesen Teil wirklich werden zu lassen, muss sich Existenz entscheiden, sie muss wählen. Mit der Wahl erhält das Abwesende des Vorgriffs deutlichere Konturen und tritt, sich im Möglichen des Projekts haltend, zugleich in den Bereich der Verwirklichung. Da Verwirklichung ein Prozess der Vergewisserung des zunächst nur Projektierten ist, richtet sich das gesamte Projektieren auf die eigene, exklusive Gewissheit aus, mit der Tendenz, Alternativen mehr oder weniger auszuschließen.

3 „In Patočka's hands, such terms as ,motion, ', existence, , essence, ', body,', soul' and ,freedom achieve fundamentally new meanings. It is in terms of these that his insistence on the unconditioned character of human rights has to be understood." (Ibid., S. 146.) 
Freiheit als Vollzug ekstatischer Bewegung bezieht Mensch nun auf Patočkas Auffassung von den drei grundlegenden Bewegungsformen menschlicher Existenz. Jede dieser Formen weise ihren eigenen Charakter der Freiheit und ihren Stil zu wählen auf. Während Freiheit in der ersten Bewegung im instinktiv-affektiven Grund der Verankerung unserer Existenz fungiert und in der zweiten sich im Feld menschlicher Arbeit auswirkt, werde sie erst in der dritten Bewegung als solche ergriffen: Freiheit wendet sich hier auf sich selbst zurück, auf die Weisen, wie sie sich in den Formen der ersten beiden Bewegungen implizit vollzieht. Mit anderen Worten: Existenz ergreift die Möglichkeit, sich zu ihrem Können selbst noch zu verhalten. Dieses neue Verhaltenkönnen zum Vollzug des Könnens bewirkt ein In-Frage-Stellen wirklicher und möglicher Handlungsvollzüge und schafft damit ein Bewusstsein des verantwortungsvollen Umgangs mit dem Vollzug des Könnens. Dazu gehöre vor allem die Einsicht, dass Gewissheiten Korrelate bestimmter Entscheidungen sind, und damit die implizite Forderung, dass jene nicht absolut angesetzt, sondern in Relation zu ihrer jeweiligen Entscheidungsgenesis gesehen werden sollten.

Die Möglichkeit, dies zu tun, umschreibe, so Mensch, der Begriff der ,Sorge', wie ihn Patočka aufnimmt. ,Sorge‘ betrifft dann den Umgang mit uns selbst, die Weise, wie wir (so oder so geworden) sind: als Verhalten zu den eigenen prätendierten Gewissheiten - als Sorge für das Erscheinen, nämlich für die Bewegungen, mit denen menschliche Existenz (,Seele') sich vollzieht und in Erscheinung tritt, sich manifestiert. Die Sorge für die Manifestationen der Existenz im Kontext ihrer Bewegungen sei der ontologische Rahmen für Patočkas Konzeption der Menschenrechte. ${ }^{4}$ Erst das Infragestellen faktischer Bewegungen menschlicher Existenz erfülle das Essentielle des Menschlichen, das darin liegt, noch die Freiheit zu haben, sich im Konkreten einer Situation seinen Bewegungen stellen zu können. Die Verwirklichung dieser Freiheit, der freien Stellungnahme zu den impliziten freien Vollzügen der sich bewegenden Existenz, erfordere jedoch ein Freisetzen von der Fixierung auf geglaubte Gewissheiten, ein leibliches Erschüttertwerden des Insistierens auf sie.

Mensch unterstreicht, dass Erschüttertwerden und das ihm möglicherweise folgende Infragestellen von selbstverständlichen Gewissheiten stets unter dem Blick des Anderen geschehen. Indem ich dabei zu ihm oder ihr in einen Bezug gesetzt werde, der nicht mehr von meinem egozentrischen Projektieren dirigiert wird, finde ich mich in einem Raum des Zwischen, der als der öffentliche oder

$4 \quad$ Ibid., S. 153. - Die Konsequenz ist eine Verankerung der Menschenrechte in der menschlichen Existenz selbst: „When Patočka asserts that human rights are not a result of our humanity - i.e., something we postulate - but rather prior to and definitive of it, his claim is that in safeguarding such rights we preserve our humanity. We do so when we care for the movements of existence that actualize us as human." (Ibid.) 
politische in einem ursprünglichen Sinn bestimmt werden kann. Das Sichhalten in diesem Raum des Infragestellens sei, mit Patočka gesprochen, „Leben in der Wahrheit ", 5 wobei Wahrheit Ausdruck der Freiheit ist, sich zu unseren impliziten freien Aktualisierungen verhalten zu können.

Die Sorge betrifft dann nicht nur die zu verantwortende Genealogie meiner eigenen Entscheidungsdispositionen, sondern umfasst die Stabilisierung des gesamten Raums wechselseitiger Verständigung über die je eigenen Gründe menschlicher Existenzen, d. h. betrifft ein Manifestieren des öffentlichen Bereichs selbst. Die Sorge für die Manifestationen der Existenz im Kontext ihrer Bewegungen als der ontologische Rahmen für Menschenrechte erfüllt sich somit erst dort, wo, wie Mensch hervorhebt, im öffentlichen Raum wechselseitiger Verständigung die Rechte freier Äußerung und freien Austauschs verwirklicht werden, ${ }^{6}$ und auch dies ist wieder wechselseitig: Durch die Etablierung eines gemeinsamen Raums der freien Rede vermag die einzelne Existenz ihre explizite Freiheit, bei der sie immer vom Anderen betroffen wird, zu verwirklichen. ${ }^{7}$

\section{Sorge und Recht}

Das hier skizzenhaft umrissene Resultat, das James Mensch zu Patočkas neuem Konzept der Menschenrechte erarbeitet hat, gibt Anlass zu weiterführenden Fragestellungen. Von diesen möglichen Fragen sei ein Komplex um das Verhältnis von Sorge und Recht herausgegriffen und in einen interkulturellen Kontext gestellt.

1. Sorge. - Bei Patočka wird deutlich, dass sich die Sorge nicht lediglich auf eine vereinzelte, ,je meine“ Existenz ${ }^{8}$ bezieht und nicht nur den Umgang mit einer ursprünglich zuhandenen ${ }^{9}$ Welt betrifft. Sowohl die erste als auch die dritte Be-

5 Vgl. ibid., S. 154.

6 „The care for this life“ - „a life ,from freedom for freedom“" (Patočka Jan, Heretical Essays in the Philosophy of History, Chicago, Open Court Publishing, 1996, p. 142) - „is the care for the soul that actualizes itself through the third motion of existence. It is, in the political realm, the care for the manifesting, the public space, that such motion makes possible" (Mensch James, Patočka's Asubjective Phenomenology..., op. cit., S. 156).

7 „For Patočka, the actualization of this openness as political freedom demands a certain framework. Viewed in terms of problematization - i.e., in terms of the third motion of our existence - it requires the rights to freely assemble, discuss, publish, petition and so on. These rights are the necessary conditions for the motion - the self-actualization - through which we achieve our full humanity. (...) Human rights, in other words, are essential for the self-directed motion that expresses our entelechia as historical and as fully human." (Ibid., S. 156f.)

8 Vgl. Heidegger Martin, Sein und Zeit, Frankfurt am Main, Klostermann, Gesamtausgabe: Bd. 2, 1977, S. 153.

$9 \quad$ Ibid., $\$ 15$. 
wegungsform zeigen, dass menschliche Existenz in einem Verhältnis zu Anderen steht, das nicht dem privativen Modus des Man ${ }^{10}$ unterliegt. Das mit der Geburt erfolgende Angewiesensein auf Andere ist nicht im Kontext einer schon zuhandenen Welt zu beschreiben, und die Möglichkeit, Öffentlichkeit im Sinne eines wechselseitigen Austauschs von Einzelnen zu realisieren, verortet sich jenseits der Alternative von verfallener und authentischer Existenz.

Die Sorge bei Patočka zielt also nicht auf die Etablierung einer Existenz, die ,ganz ${ }^{11}$ nur im angeeigneten Eigenen wird, sondern die sich gegenüber Bewegungsformen menschlicher Existenz und ihren jeweiligen Aktualisierungen in Stellung bringt. Hier ist kein Ende in Sicht, kein Vorlaufen in den je eigenen Tod, kein Ganzwerden, ${ }^{12}$ sondern im Gegenteil die Relationierung eines Totalitätsanspruchs, der mit der Projektion der eigenen Wahl absolut gesetzt wird; und dieser Abbau der selbstverständlichen Gewissheit ist eine unablässig zu unternehmende, nicht abschließbare Aufgabe, da die Freiheit, dies zu tun, Freiheit ebenfalls nur in Bewegung ist.

Mit dieser Auffassung stellt sich Patočka in eine Reihe zu anderen Autoren und bereichert das Profil, das man bei ihnen jeweils vorfinden kann. Dieser Austausch zwischen philosophischen Positionen in der Absicht einer wechselseitigen Bereicherung ist selbst das Beispiel einer sich im Sinn von Patočka vollziehenden dritten Bewegung. Formal gesprochen geht es dabei um das Verhältnis von Teil und Ganzem, wobei vermieden werden soll, den Teil als Ganzes und das Ganze ganz zu denken. Dies hatte vielleicht schon Heraklit im Sinn, wenn er die sich in sich verschließenden Meinungen (,Gewissheiten') der Einzelnen mit deren Öffnung konfrontierte. ${ }^{13}$,Logos' mag dabei lediglich Ausdruck einer Öffnung sein, wobei Heraklit es tunlichst vermied, das allen Realisierungsformen des Öffnens gemeinsame Prinzip eindeutig zu bestimmen. Das Unbestimmte des Logos ist eine Schutzfunktion vor seiner Substantialisierung und Absolutsetzung, wenn das, was das je Eigene übersteigt, auf eben dieses Eigene appliziert und verabsolutiert wird. Logos' ist dann nur das meontisch ${ }^{14}$ angesetzte Ganze, das ,Ganze', das im Versuch,

$10 \quad$ Ibid., $\$ 27$.

11 Ibid., $\$ 47 \mathrm{f}$.

12 Ibid., $\$ 53$.

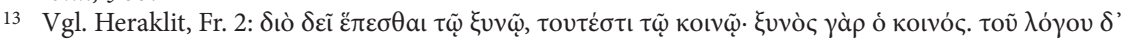

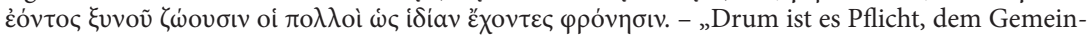
samen zu folgen. Aber obschon der Sinn gemeinsam ist, leben die Vielen, als hätten sie eine eigene Einsicht." (Diels Hermann, Kranz Walther (Hrsg.), Die Fragmente der Vorsokratiker, griechisch und deutsch: Bd. 1, Dublin/Zürich, Weidmann, 1966, S. 151)

14 ,Meontisch' ist, in Anlehnung an Eugen Fink, solches, das sich als Anwesenheit eines, ganz' Anderen, mich Übersteigenden im Horizont meines Verstehens lediglich im nicht seines permanenten Ausstands zeigt. 
es positiv zu fassen, sofort entgleitet; es ist nur der Ausdruck, dass das je einzelne nicht alles ist.

Freilich ist es nicht beiläufig, dass es der Logos ist, der dafür einstehen soll. Platons Absicht wird es bekanntlich sein, dass, mit dem meontischen nescire des Sokrates im Hintergrund, es darum geht, Freiheit in einem gemeinsamen dialege-

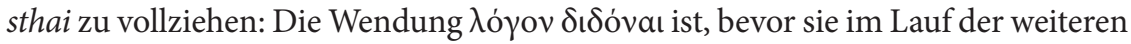
Denkgeschichte zur Darlegung von Gründen simplifiziert wurde, eine Bewegung menschlicher Existenz, deren Freiheit auch hier Wahrheit fundiert. Der Rekurs auf den gemeinschaftlichen Dialog, durch die Rede Möglichkeiten und Grenzen existenzieller Gründe darzulegen, ist offenbar das Verfahren, das Platon nutzte, um der Totalisierung partieller Existenzprojekte vorzubeugen. Die Verwirklichung dieser Freiheit, im dialegesthai die Grenze des Eigenen im Angesicht des Anderen zu suchen, wird Hans Lipps mit dem Ausdruck „sich betreffen bei“ zu fassen suchen: nämlich die Freiheit zu ergreifen, das eigene Projektieren nicht einfach zu vollziehen, sondern sich bei ihm zu betreffen. ${ }^{15}$ Das Projektieren des Hinausstehens, womit sich Existenz in Welt findet und von der Bewegung seiner egozentrischen Natur aus seine Umwelt an sich anpasst, hat schon Max Scheler mit der griffigen Formel beschrieben, dass das Ich der natürlichen Weltanschauung sein Umfeld zunächst für die Welt überhaupt nimmt. ${ }^{16}$

James Mensch verweist in diesem Zusammenhang auf das von Husserl beschriebene Verhältnis von Intention und Erfüllung: Die faktische Intention ist Resultat einer Wahl, und da sie ,ganz' auf Erfüllung aus ist, tendiert Existenz dazu, sich ganz in diese Erfüllbarkeit zu verlegen. Gewissheit als Widerschein oder besser: Vorschein dieser projektierten Erfüllung verfügt über die Kraft, die sie tragende Intention absolut zu setzen. Die Betonung, die der spätere Husserl auf den Aufweis von Wahrheitsgeschehen legt, ist Ausdruck dafür, die Verantwortung für ,Wahrheit' nicht erst beim Endprodukt beginnen zu lassen, sondern auf seine Ge-

15 Wer Epoché übt, „betrifft sich bei“ seinem Grund; auf diese Weise werde er „freigemacht von der Befangenheit in sich selbst. Daß er betroffen wird bei... bedeutet (...) eine Unterbrechung = epoché. Und weil Existenz durch andere Existenz dahinversetzt werden kann, ist Philosophie selbst ihrer Möglichkeit nach Problem geworden, wenn die Verhältnismäßigkeit des dialégesthai untersucht werden soll. Im freien Verhältnis des einen zum anderen geschieht Philosophieren" (Lipps Hans, Untersuchungen zu einer Hermeneutischen Logik, Frankfurt am Main, Klostermann, Werke: Bd. II, 1976, S. 22, Anm.).

16 „Unter Egozentrismus verstehe ich die Illusion, die eigene ,Umwelt‘ für die ,Welt` selber zu halten, d. h. die illusionäre Gegebenheit der eigenen Umwelt als ,die' Welt.“ (Scheler Max, Wesen und Formen der Sympathie, Bern/München, Francke, Gesammelte Werke: Bd. 7, 1973, S. 69) Dies meint nicht einfach den Vorzug von Sinngehalten der eigenen Weltanschauung, sondern die Privilegierung desjenigen Erlebens, für das diese Inhalte je Gültigkeit haben: Die Orientierung des solcherart welterfahrenden Lebens verläuft von diesem aus auf es selbst zurück. 
nealogie, seine Bewegung, zu beziehen. Mit Levinas gesprochen, wäre dies das Eintreten für ein unendliches Begehren, das sich nicht in den Gewissheiten eines sich verendlichenden Begehrens festmacht. ${ }^{17}$

Bei alldem geht es nicht mehr um den Bezug auf Ganzheiten, seien dies Substanzen, Subjekte oder Objekte. Der Paradigmenwechsel, der nicht nur mit der Phänomenologie erfolgt, verlegt sich vom Terminieren im Teleologischen entschieden auf das „Teleologischsein“. ${ }^{18}$ In der Terminologie von Ganzem und Teil gesprochen bedeutet dies, dass es um die Relation der Teile geht, wobei das, was nicht mehr einen Teil ausmacht, als seine meontische Grenze ihm noch zugehört. Diesen meontischen Bezug fasste Eugen Fink mit dem Begriff des symbolon in seinem ursprünglichen Sinn als Bruchstück. ${ }^{19}$ Menschliche Existenz ist unablässig in Bewegung, da sie Fragment ist. In gewissem Sinn wusste dies eben schon Platon. ${ }^{20}$

2. Europa. - Das Gesagte betrifft jedoch nur das europäische Denken - so zumindest könnte es den Anschein haben. Und in der Tat hat dies ja Patočka selber betont. Die dritte Bewegung der menschlichen Existenz, die Problematisierung ihrer eigenen Freiheit, sei, wie er in den Ketzerischen Essays ausführt, durch Philosophie und Polis in die Welt gekommen. ${ }^{21}$ Wenn sich die Menschenrechte zwar in allen drei Bewegungen menschlicher Existenz manifestieren, in der dritten jedoch erst als solche formulierbar werden, sind sie zunächst an denjenigen Ort gebunden, an dem der faktische Durchbruch dieser Bewegung erfolgt: eben an Europa. Nun ist gewiss zu unterscheiden zwischen dem faktischen Ort und der faktischen Zeit, an dem und zu der ein Mögliches sich aktualisiert, einerseits und diesem Möglichen selbst andererseits. Dass eine Möglichkeit faktisch ergriffen wird, bindet sie nur in einem gewissen Sinn an den Vorgang des Ergreifens, wird aber nicht relativ auf ihn. Auch hat, was die Bewegung des Durchbruchs menschlicher Existenz, des

17 Vgl. Levinas Emmanuel, Totalité et infini. Essai sur l'extériorité, La Haye, Nijhoff, Phaenomenologica: Bd. 8, 1961.

18 Husserl Edmund, Die Krisis der europäischen Wissenschaften und die transzendentale Phänomenologie, Den Haag, Nijhoff, Husserliana: Bd. VI, 1954, 275.

19 Fink Eugen, Spiel als Weltsymbol, Freiburg/München, Karl Alber, Eugen Fink Gesamtausgabe: Bd. 7, 2010, S. 122.

20 Sowohl Platons Gastmahl als auch der Bericht darüber sind eine Versammlung von Bruchstücken. Der Grundgedanke des im Symposion sich manifestierenden symphilosophein ist das Zusammenkommen von Individuen, die nur deshalb ein Gemeinsames, ein Zwischen (syn-) realisieren können, weil sie selbst je ein völlig Abgesondertes (in-dividuum) sind. Indem diese ab-soluten ,Teile' (Fragmente, sýmbola im Sinn der tesserae) sowohl dasjenige bleiben, was sie sind, als dies auch den Anderen zugestehen zu sein, vollzieht sich das sýn. - Vgl. hierzu ausführlicher v. Vf.: Sepp Hans Rainer, „Der Gast als Symbol“, in Liebsch Burkhard, Staudigl Michael, Stoellger Philipp (Hrsg.), Perspektiven europäischer Gastlichkeit. Geschichte - Kulturelle Praktiken - Kritik, Weilerswist, Velbrück Wissenschaft, 2016, S. 426-449.

21 Patočka Jan, Ketzerische Essays zur Philosophie der Geschichte, Berlin, Suhrkamp, 2010, S. 47 ff. 
Freiwerdens zur Freiheit, betrifft, Europa mit Philosophie und Polis nicht einen Masterplan für die gesamte übrige Welt und für alle Zeiten aufgestellt. Die Frage würde also lauten, ob sich diese Möglichkeit nicht in anderen Kulturen zu anderen Zeiten ebenfalls aktualisiert hat, wenngleich nicht in der Art und Weise, wie dies in Europa geschah. Könnte diese Frage mit Ja beantwortet werden, erwächst die Aufgabe, dies zu belegen, indem Weisen des Selbstverhaltens menschlicher Existenz in den Grundmodi ihres Vollzugs befragt und darüber hinaus Unterschiede der Verwirklichung dieses Möglichen aufgezeigt würden.

3. Interkulturelle Perspektiven. - Parallele Erfahrungen zeigen sich in Ostasien. Nimmt man nur Formen des Buddhismus im Allgemeinen, des Zen-Buddhismus im Besonderen in den Blick, so stößt man auf ähnliche Strukturen. Im Vordergrund steht auch hier die Überwindung egozentrischer Einstellungen, also des Hinausstehens in je eigene Gewissheiten. Diese Überwindung erfolgt hier jedoch nicht im Zuge eines gemeinschaftlichen dialegesthai, wo je ,private Auffassungen miteinander konfrontiert und in ihrer jeweils absolut gesetzten Glaubensrelation erschüttert werden. Sie geschieht in einem absolut vereinzelnden Erlebnis wie dem Satori - doch zugleich im Kontext desselben Zwecks: die einzelne Existenz aus ihrem egozentrischen Bezug zur Welt zu lösen und sie im Rückgang auf ihr einzigartiges, von keinem Anderen zu übernehmendes Erleben in den offenen Kontext eines sie Umfassenden zu stellen. Das Resultat, ein verändertes Verhältnis zur Welt, wird auch hier meontisch beschrieben: nur durch die Realisierung des Nichts (der Preisgabe der egozentrischen Verankerung) einen Zugang zur offenen Fülle des Wirklichen, die nicht mehr auf die private Gewissheit relativ ist, zu erlangen. ${ }^{22}$

Die Bewegung des Rückgangs besitzt Parallelen in der ausgehenden europäischen Antike, insbesondere im Christentum, beispielsweise im in te redi des Augustinus, das noch Husserl als Motto seines Anliegens dienen sollte. ${ }^{23}$ Zugleich macht der Hinweis auf eine absolut vereinzelnde Aktualisierung darauf aufmerksam, dass die Kehre menschlicher Existenz noch nicht dadurch hinreichend bestimmt ist, dass von einer Sorge gesprochen wird, die sich auf den Vollzug ihrer Bewegungsmöglichkeiten bezieht. Denn die grundsätzliche Frage lautet, wie die Aktualisierung der Freiheit gegenüber den freien Aktualisierungen erfolgen kann. Was löst die Unterbrechung der fortlaufenden Verlängerung in die eigene Projektion hinein aus, und wie erfolgt diese „Pause des Lichts“, wie Aldo Rovatti es einmal formuliert

22 Vgl. z. B. Ueda Shizuteru, „Leere und Fülle - Sunyata im Mahayana-Buddhismus. Zum Selbstgewahrnis des wahren Selbst", in Ueda S., Wer und was bin ich? Zur Phänomenologie des Selbst im Zen-Buddhismus, Freiburg/München, Karl Alber, 2011, S. 11-37.

23 Husserl Edmund, Cartesianische Meditationen und Pariser Vorträge, Den Haag, Nijhoff, Husserliana: Bd. I, 1950, S. 183. 
hat?24 Im Kontext phänomenologischen Denkens wurden hierzu, wo es um die Praxisseite des Epoché-Geschehens geht, nur Andeutungen gegeben, etwa bei der Frage nach der Motivation der phänomenologischen Reduktion, auf die Fink in der VI. Cartesianischen Meditation mit dem Hinweis auf "Grenzsituationen“ menschlicher Existenz, in denen die Tendenz zu einem kontinuierlichen Fortschreiten der Welterfahrung gebrochen werden kann, eine vorläufige Antwort formuliert. ${ }^{25}$ Ein Pendant dazu ist die von Patočka später namhaft gemachte „Erschütterung“ ${ }^{26}$ die wiederum in diesem Kontext zu lesen ist. Das wichtige Moment, das man dieser Problematik entnehmen kann, liegt darin, dass die gemeinschaftliche Garantie der Menschenrechte allenfalls einen Rahmen schafft, in dem sich die Freiheit zur Freiheit existenzieller Aktualisierungen vollziehen kann, nicht aber den Vollzug selbst bestimmt, der stets individuell und individuierend ist.

Die Befreiung zum dialegesthai ist somit ein kritischer, ein gefährdeter Bereich, das Nadelöhr, durch das die einzelne Existenz hindurch muss und das durch kein Recht allein zu stützen ist. Die Freiheit zur Freiheit ist nicht aus dem Horizont der Welt ableitbar, da sie gleichsam vertikal zu ihm steht und ihn als solchen betrifft; sie muss von der einzelnen Existenz ergriffen werden, ohne dass dies nur ein Rückzug in die Einsamkeit des Egos wäre. Wie Patočka betont und Mensch nachdrücklich hervorhebt, ist dieser Rückgang durch die Konfrontation mit Anderen veranlasst und hat in der Bewegung auf diese hin auch ihr letztes Ziel. Ab-soluter, sich von der Fixiertheit auf je eigene Gewissheiten ablösender Rückgang ins Selbst und erneuerter Bezug zu Anderen sind also vor dem Hintergrund sowohl eines beständigen Bezugs des Einzelnen zu allem Anderen als auch der ab-soluten, separierten Existenz des Einzelnen zusammen zu denken. Hierin besteht gewiss der Ort für die Aktualisierung grundsätzlicher menschlicher Rechte, und sollte es diesbezüglich in der Tat parallele Erfahrungen in anderen Kulturen geben, wäre ein Spalt geöffnet, in einem interkulturellen dialegesthai diese Formen des Aufbruchs einander näher zu bringen und die Rede von Menschenrechten transeuropäisch zu verankern.

Ein weiteres Beispiel bietet die Kultur der australischen Aborigines. Es verdeutlicht, dass es Gemeinschaften gegeben hat und evtl. noch gibt, die durch die Praxis eines ursprünglichen, den grundlegenden Bewegungen menschlicher Existenz unmittelbar entnommenen Verständnisses vom Recht der Einzelnen versuchen, das

24 Rovatti Pier Aldo, „Das Rätsel der Epoché“, in Jamme Christoph, Pöggeler Otto (Hrsg.), Phänomenologie im Widerstreit. Zum 50. Todestag Edmund Husserls, Frankfurt am Main, Suhrkamp, 1989, S. 277-288; hier: S. 278.

25 Fink Eugen, VI. Cartesianische Meditation. Teil 1: Die Idee einer transzendentalen Methodenlehre, Dordrecht/Boston/London, Kluwer, Husserliana Dokumente: Bd. II/1, 1988, S. 38.

26 Vgl. z. B. Patočka Jan, Ketzerische Essays, op. cit., S. 141 ff. 
Aufkommen egozentrischer Strukturen von vornherein zu verhindern. ${ }^{27}$ Denn in ihrer tradierten Weltsicht verhalten sich Aborigines im Gegensatz zu anderen Sozietäten zu ihrem Land nicht wie zu einem Gegenstand, von dem sie Besitz ergreifen, den sie aufteilen und als ein zu nutzendes Reservoir für Lebens- und Machtmittel verwenden, um das es zu kämpfen gilt, ${ }^{28}$ sondern es ist vielmehr umgekehrt das Land, das das Verhalten der Menschen bestimmt. ${ }^{29}$ Diese Bestimmung steht von vornherein in einem intimen Bezug zum Menschen, indem das Land für ihn nicht erst dort eine Rolle spielt, wo er oder sie dem Land als Handelnde gegenübertreten; schon Zeitpunkt und Ort der erstmals ,Eintritt' in das Land verschaffenden Geburt - oder bereits der Zeugung bzw. auch erst der ,zweiten Geburt', der Initiation, - verleihen ihm oder ihr einen rechtlichen Status und Anspruch. Das Recht leitet sich somit nicht von den Setzungen einer Gruppe her, etwa von der aus einer Ansiedlung entsprungenen Kodifizierung. Da jeder Mensch geboren wird, ist das ,Land-Recht ${ }^{\text {' }}$ sowohl das Recht auf Land als auch das Recht selbst in seinem Ursprung aus dem Bezug zum Land - für alle gleich.

Darin liegt ein Mehrfaches: 1. zum einen eine Egalität, sofern nicht nur alle ,von Geburt aus gleich' sind, sondern für alle grundlegender noch das schlichte Faktum relevant ist, überhaupt geboren zu sein; 2. des Weiteren eine als selbstverständlich empfundene Pflicht gegenüber dem ,Verliehenen', was eine Pflege des Übergebenen bedeutet, die sich in einem besonderen Wissen konkretisiert. Diese Pflicht resultiert sowohl aus dem Faktum der Geburt wie auch aus dem Verhältnis zum Land, zu Orten, welche die Ahnen gestiftet haben, und zu solchen, die in der Lebensspanne von Geburt zu Tod eines Menschen neu markiert werden.

3. Ein spezifisches Verhältnis zwischen absolut und relational. Während Formen des Naturrechts wie des positiven Rechts auf einen Bereich verweisen, der in seiner Legitimation und Geltungsfunktion den einzelnen Rechtspersonen übergeordnet ist, ist das aus dem Zueinander von Geburt und Land entsprungene Recht

27 Das soziale Modell der australischen Aborigines ließe sich noch mit Patočkas dreistufiger Gliederung der Bewegungen menschlicher Existenz beschreiben und zeigen, dass es insofern einen extremen Fall darstellt, als hier durch eine bestimmte Erziehungspraxis (im Kontext der ersten Bewegung) die zweite Bewegung vermieden und damit die dritte Bewegung obsolet wird. Es wäre falsch, daraus eine Relativität von Verwirklichungsformen existenzialer Freiheit und von Rechtskonzepten zu folgern; vielmehr geht es darum, Realitäten zu beschreiben: aufgrund ihres jeweiligen Weltzugangs gegeneinander nicht aufzurechnende Formen eines sowohl individual als auch sozial ,glückenden, durch die Bändigung des sich in seinen Gewissheiten verfangenden Egos gekennzeichneten Lebens.

28 „We never fought over ground, we never fought over territory.“ (Meenamatla Everett, „Ältester des Plangermairreenner Clan, Ben Lomond Tribe, Tasmanien“, in Elders. Wisdom From Australia's Indigenous Leaders (photographed and recorded by Peter McConchie), Cambridge, Cambridge University Press, 2003, S. 58.)

29 "The Law of the Land and our responsibilities, they were laid down by the land itself." (Ibid.) 
lediglich darin allgemein, dass seine Bestimmungen, vor dem Hintergrund eines gemeinschaftlichen religiösen Wissens, ${ }^{30}$ mit der (ersten oder zweiten) Geburt einer und eines jeden, aktiviert' werden. Dabei gilt zugleich, dass der Geltungsbereich einer Person seine Grenze am Geltungsbereich einer anderen findet und somit diesen respektieren muss, so wie erwartet werden kann, dass auch er oder sie respektiert werden. Der Rechtsanspruch gründet somit absolut in jeder oder jedem kraft ihrer oder seiner Geburt, wie er zugleich vom Faktum anderer Einzelner oder Gruppen relativiert wird. Die Einzelnen sind unabhängig nur bezüglich des leiblichen Befunds der Tatsache ihrer Existenz und der darin verankerten (vor dem Hintergrund des gemeinsamen religiösen Wissens) je einzigartigen spirituellen Bedeutung; sie sind zugleich abhängig, sofern sie auf alle Anderen, die in ihrem Ursprung ebenso unhintergehbar sind, angewiesen bleiben. In diesem ursprünglichen Sinn steht der einzelne Mensch im Zwischen eines Absoluten (Herausgehobenen) und einem Relationalen (Eingebundenen), jedoch nicht in dem Sinn, dass er im Durchschnitt von beidem lebt, sondern mit seiner Existenz beides austrägt.

Daraus resultiert 4. eine eigentümliche Perspektivik: Das Land ist Träger für ein Netz von Verbindungen zwischen Personen, zwischen einer Person und ihrer Gruppe, zwischen dieser und anderen Gruppen. Sofern mit der Geburt eine einzelne Existenz und das Land untrennbar zusammen treten, schafft das Land die Matrix, auf deren Grund sich die Bewegungen aller Beteiligten einzeichnen, so jedoch, dass jede oder jeder nur einen, aber je irreduziblen Abschnitt dieser Grundmatrix realisiert. Wissen und Recht sind auf diese Weise geteilt und als je einzigartig und nicht ersetzbar zugleich auf Ergänzung angelegt. In der Ausgestaltung des Wissens verknüpft sich die Faktizität einer und eines jeden mit dem Ahnenwissen der betreffenden Gruppe. In einer Gruppe treten Älteste (elder persons) hervor, die bis zu einem gewissen Grad über das Wissen, das eine Gruppe betrifft, verfügen, doch auch dieses Wissen wertet ihren sozialen Status gegenüber ihren Gruppenmitgliedern nicht auf, sie sind Bewahrer und stehen nicht einer Gruppe vor. Diese Grundstrukturen haben zur Folge, dass weder zwischen Einzelnen und der Gruppe noch zwischen Gruppen untereinander das Bestreben vorherrscht, kulturelle Grenzen definitiv zu ziehen. ${ }^{31}$ Einzelne und Gruppen sind vielmehr auf Möglichkeit und Notwendigkeit der Vertiefung ihres Wissens durch

30 Dieses Wissen beinhaltet vor allem Schöpfungsmythen, wobei es, trotz der Vielfalt der australischen Ethnien, einen Kern von gemeinsamen Inhalten und eine davon abweichende Variabilität gibt. Erstaunlich ist, dass sich dieser Kern über Raum und Zeit hinweg durchhält, nimmt man noch hinzu, dass bei der ausschließlich mündlichen Weitergabe es Brauch ist, Änderungen und Ausschmückungen vorzunehmen.

31 Einzig dem Land kommt es zu, Grenzen zu ziehen: „The land had created the boundaries (...).“ (Ibid.) 
Austausch angelegt, was insbesondere in periodisch wiederkehrenden Festen realisiert wird.

Die Möglichkeit, Verbindungen zu knüpfen, besitzt ihren Ursprung im Zusammentreten von Geburt und Land bzw. im Zwischen von beidem: wie der Mensch nicht mehr hinter seine Geburt zurück kann, so kann er auch nicht ganz im Land aufgehen. Eben dieses nicht spannt ein Verwirklichungsfeld als ein Zwischen auf, das für das Zustandekommen der Verbindungen aufkommt. Dabei wird dieses Zwischen durch zwei Grenzbereiche stabilisiert: Die eine Grenze bildet das Geschehen der Geburt, in dem sich das Zusammentreten von Geburt und Land ereignet, die andere Grenze ist durch die Möglichkeit bezeichnet, das eigene stets begrenzte Wissen wiederholend zu bewahren, es aber auch auszuweiten und dabei das Wissen Anderer aufzunehmen, was ebenfalls in Verbindung zum Land, zu Orten, erfolgt. Beide Grenzbereiche fluktuieren, sofern das Geschehen der Geburt nicht lediglich ein Faktum der Vergangenheit darstellt, sondern in jeder Gegenwart weiter wirkt, und die Möglichkeit zu künftiger Ergänzung und Veränderung des Wissens prinzipiell unbegrenzt ist und Gegenwart ebenfalls mitbestimmt.

Bezüglich der Bewegung der Existenz können mithin zwei aufeinander sich aufstufende und dabei ineinander wirkende Ebenen des Zwischen unterschieden werden: 1. das Zwischen von Geburt und Land und dies als Matrix für 2. dasjenige Zwischen, das sich zwischen Personen und Gruppen aufspannt, indem Einzelne sich zu ihrer Herkunft verhalten, aber zugleich auch zu (den Orten von) Anderen in Beziehung setzen und so das eigene Wissen mitteilen und selbst einen Wissenszuwachs erhalten. Vollzieht sich Leben als Ineinander beider Ebenen, ist die zweite, in die Matrix sich einschreibende Bewegung die eigentliche Vollzugsdimension: Die Relation von Geburt und Land bildet ein relativ statisches Moment, das auf der zweiten Ebene der konkreten Daseinsvollzüge in eins mit diesen in eine Dynamik hineingenommen ist. Da diese Dynamik von der Spannung des Gegensätzlichen getragen wird - sofern weder Geburt und Land noch die Einen und die Anderen zu einer Einheit tendieren -, verwirklicht sich das Zwischen von Geburt und Land so, dass bezüglich der einzelnen Existenz das Verhältnis zu sich selbst und zu Anderen lebendig bleibt. Dies kann auch so formuliert werden, dass es darum geht, auf der Grundlage des Landes als eines Allgemeinsamen und als solchen nicht Teilbaren sowie der Unhintergehbarkeit der eigenen Geburt sich derart in einem Zustand des (Mit-) Teilens zu halten, dass die eigene Verortung weder dominiert noch preisgegeben und dementsprechend der Bezug zum Anderen weder beherrschend noch unterwerfend ausgestaltet wird.

Auf diese Weise die Spannung zwischen der eigenen Geburt und den Orten der Anderen auf der Grundlage des Landes zu halten, stabilisiert das Netz der zu- 
stande kommenden Verbindungen, und zwar so, dass dieses Netz prinzipiell offen ist und damit keine ein- und abgrenzenden Ränder besitzt. Dies betrifft auch das Verhältnis von geheimem Wissen und seiner Äußerung. Das mit Abstammung und Geburtsort sowie weiteren besonderen Stätten verbundene Wissen ist nicht ohne weiteres mitteilungsfähig; andererseits gibt es Wege, es mitzuteilen. Der bildliche Ausdruck ${ }^{32}$ mag einer dieser Wege sein, der äußert, was vielleicht durch Worte nicht gesagt werden kann oder darf. Autorin oder Autor eines Bildes zu sein, bedeutet daher auch, ein Zwischen in diesem weiteren Sinn auszutragen, wenn es - neben der Spannung von Geburt und Land, dem Recht und dem Wissen Einzelner und aller Anderen -, auch darum geht, sich im Zwischen von Geheimnis und Äußerung zu halten. ${ }^{33}$

Wie dieses Beispiel der Aborigines vorführt, scheint es ein durchaus sinnvolles Unternehmen zu sein, Grundbewegungen menschlicher Existenz in anderen kulturellen Kontexten herauszuarbeiten. Das Bemerkenswerte ist, dass diese Bewegungen mit ebenso basalen Formen des Rechts als ,Menschen-Recht' verbunden sind, wie James Mensch dies exemplarisch an der von Patočka vor dem Hintergrund europäischer Denk- und Lebensgeschichte vorgenommenen Analyse der Bewegungen menschlicher Existenz aufgezeigt hat. Ein interkulturelles dialegesthai über die existenziell verortete Dimension der Menschenrechte wird nicht umhin kommen, die in den Bewegungsformen menschlicher Existenz jeweils verankerte Rechtsauffassung darzulegen, um das Bestehen von Menschenrechten im Kontext ihrer kulturellen Abschattungen ausweisen zu können. Einen ersten wichtigen Schritt in diese Richtung, Menschenrechte und Bewegungen der menschlichen Existenz ab ovo zusammenzudenken und Wege zu eröffnen, über Europa hinauszudenken, hat James Mensch mit seinem Buch zu Patočka unternommen.

Hans Rainer Sepp teaches philosophy at Charles University, Faculty of Humanities and shares the directorship of the Central-European Institute of Philosophy (Středoevropský institut pro filosofii), with Karel Novotný.

E-mail: hr.sepp@web.de

32 Zum Kunstschaffen der Aborigines vgl. Caruana Wally, Die Kunst der Aborigines, München, Lichtenberg, 1997; McCulloch Susan, McCulloch Emily, Contemporary Aboriginal Art, Melbourne, McCulloch \& McCulloch, 2012.

33 Hierzu ausführlicher v. Vf.: „Diesseits von Heimwelt und Fremdwelt. Die Weltstruktur der Aborigines ein Sonderfall?", in Beckman-Zöller Beatte, Kaufmann René (Hrsg.), Heimat und Fremde. Präsenz im Entzug. Festschrift für Hanna-Barbara Gerl-Falkovitz, Dresden, Text \& Dialog, 2015, S. 337-354. 